\title{
A New Approach to Communications Management Planning Through 3D Web and Semantic Web Technologies
}

\author{
Charles J. Lesko, Jr. (Corresponding author) \\ College of Technology \& Computer Science, East Carolina University \\ Science \& Technology Building, Suite 212, Greenville, North Carolina 27858 \\ Tel: +1-252-737-1907Ｅ-mail: leskoc@ecu.edu \\ Yolanda A. Hollingsworth \\ East Carolina University \\ Joyner Library, Greenville, North Carolina 27858 \\ Tel: +1-252-328-6518Ｅ-mail: hollingsworthy@ecu.edu
}

Received: July 6, $2011 \quad$ Accepted: July 28, $2011 \quad$ doi:10.5430/jms.v2n3p25

\begin{abstract}
Communication management plans are used to determine not only who needs what information but also how that information will be collected and transmitted. Now two evolving technologies are looking to drive project planners to develop new approaches and methods for planning communications in the coming years. The first of these technologies, the Semantic Web, is becoming a driving force in how computers are making web content available to its users. The second technology, Web three-dimensional (3D) focuses on web-based content presentation by providing a rich 3D Web-centric environment for users to access information and interact with other users. This effort discusses the advent of the Semantic Web and Web 3D technologies and identifies many of the new planning considerations driving project information collection and analysis. The planning considerations for these two technologies are also discussed to aid in the framing of a new approach to project communications planning.
\end{abstract}

Keywords: Communications Planning, Semantic Web, 3D Web, Project Management, Virtual Worlds

\section{Introduction}

It has often been said that a project manager's most important skill is their ability to communicate. But in order to assure successful delivery of any project, it is crucial for all project team members and stakeholders to accurately inform each other of their needs, updates and timelines - in short, they need to communicate. To ensure timely and meaningful exchange of information today's project communication management plans rely heavily on Internet or Web-based communications in the form of email, blobs, tubes, websites, and other web-based services (Ollus, Jansson, Karvonen, Uoti, \& RiiKonen, 2009) (Farr \& Irias, 2008). The vast usage of web-based communications is shifting the planning focus from a more centrally controlled approach to communication planning where media and content resources are more distributed, empowering, and engaging to all involved. Today's project leaders are also being challenged to embrace and plan for many new and evolving web-based technologies.

Over the past two decades the World Wide Web (WWW) saw a 1990's era, often referred to as Web 1.0, that focused mostly on read-only content and static HTML-based websites; early websites were generally not interactive as the Web technologies concentrated primarily on linking documents across the Web. Web users over the past ten years shifted gears with web service and technologies that have focused heavily on user-generated content. This recent use, often been referred to as Web 2.0 or the Social Web, has proved users with more of a read-write Web capability where users have been contributing as well as consuming Web content through the use of forums, blogs and social sites like Twitter, MySpace, YouTube, and Facebook (Strickland, 2009) (O'Reilly, 2005). In a time when the Web enables all its users to be Web publishers, project managers are witnessing an explosion of content that is being exchanged between team members and stakeholders, up and down both formal and informal channels of communications.

These trends of social interaction, auto-content generation, are all lead-in's to what is often referred to as the Web 3.0 or the Semantic Web. The underlying premise here is that more meaning to the web content will bring about more 
intelligent searches and a more personalized experience for web users (Agarwal, 2009). At the crux of the issue here is that while computers understand the syntax of what to retrieve computers do not understand the meaning or semantics behind the content they are presenting web users. Building a Semantic Web is akin to creating a collectively intelligent and globally linked database from the Web in such a way as to be easily processed by the computer systems that maintain it with the overarching goal of providing a more efficient means of retrieving the data.

From a historical perspective, the original WWW was designed as an information space, with the goal that the Web should be useful for not only human-to-human communication, but also machine (computer) communications. But there is a major obstacle here, that while computers understand the syntax of what to retrieve computers do not understand the meaning or semantics behind the content they are presenting web users. Today's search mechanisms find information on the web largely through search words or phrases. The challenge with the majority of data on the Web is that in its current form it is difficult to use on a large scale.

A second web-based technology is evolving to a point where its inherent advantages as a means of presenting information are becoming more apparent. Continued interest and calls for more collaborative technologies has grown across professional arenas from researchers, business communities and social networks alike (Masinsin, 2008). In recent years, the use of 3D Web environments has seen tremendous growth to a point where the Web now supports nearly 580 million users worldwide with nearly half of those falling into the up and coming 10-15 year old age group (K Zero, 2009). Current reports also show nearly 150 3D virtual environments in existence today with that number expected to grow to 900 or more within the next three years (Mitham, 2009).

As a 3D Web interface, these environments provide users with some unique capabilities. Virtual Worlds Review (2009) cites six distinct functionalities that all 3D Web virtual environments have in common: First is the concept of 'shared space' where the world allows multiple users to participate at once. Secondly, there is the use of a 'graphical user interface' where the environment depicts space visually. Third, there is a sense of 'immediacy' where interaction takes place in real time. Fourth, the environment provides for interactivity allowing users to alter, develop, build, or submit customized the content. Fifth, there is a level of persistence in that the world's existence continues regardless of whether users are logged in. Finally, the sixth distinct functionality is the environment facilities the concept of socialization and community allowing and encouraging the formation of in-world social groups (Virtual Worlds Review, 2009).

From a functional perspective, early 3D Web efforts have taken advantage of Web 3D technologies in many areas including social presence, persistence and the visual presentation of the environment. The early emphasis has been centered on building out these environments and providing for a growing demand for more social-context and interaction; however, there are some recent indicators that show a growing interest in the use of virtual 3D Web environments as vehicles for presenting content (Burden, 2009).

To understand how to manage these two evolving technologies a brief discussion of current communication planning approaches and methods is covered. That discussion is followed by an assessment of the need to change and the identification of where the 'wins' are for the different stakeholder perspectives. Final discussion offers some new approaches to planning communications in consideration of these new two technologies.

\section{Current Approaches to Communications Planning}

An essential part of the project team planning efforts focuses on the communicating processes from both an internal project team perspective as well as external perspective and includes all project relevant communications between stakeholders. Management of project communications begins with an understanding of who needs what information when and generally includes all aspects of generating, collecting, disseminating, and storing communications (Phillips, 2006). But today's communication challenges are taking on a new character with the ever-increasing use of various Web-based online resources. Ease and availability of Web-centric communication modalities make documenting the processes, identifying and monitoring and retaining project-specific content and managing expectations of project communications complex. Conventional project communications planning incorporates several key areas of consideration including: (1) identifying stakeholders; (2) planning communications with respect to who gets what information when; (3) distributing information; (4) managing expectations; and (5) reporting performance (Schwalbe, 2010) (Nicholas \& Steyn, 2008).

\subsection{Identifying Stakeholders}

Individuals who hold any stake or claim to a given project; each are unique in many respects. As individuals stakeholders maintain specific roles and responsibilities not only to their parent organization but also to the project itself. Beyond documenting each stakeholders contact information, roles and relationships (both on and off the project), knowledge of 
their level of interest and influence with respect to the project and a thorough understanding of what information each stakeholder requires are considered key to communications planning.

\subsection{Planning Communications}

Over the past two decades innovations in multimedia presentations and content distribution have witnessed significant shifts in project management approaches and planning methodologies that influence communications direction and flow within a given project. Several approaches to planning their communications are currently available to project leaders and include:

- Systems Approach - This approach to communications planning finds its basis within general system theory; the theory espouses that to understand fully the operation of an entity (in this case project-centric communications), the entity must be viewed as a system. (Kerzner, 2006).

- Structural Approach - This approach uses models to capture and make visible the structure of complex projects. The structural approach has particular value for the management of projects in which the task structure is complex, uncertain, and unstable, as is typically the case in new product development type projects (Harrison \& Lock, 2004).

- Managerial Approach - The managerial approach follows the underlying premise that projects have more similarities than differences, and thus the performance of all projects can be optimized by the application of established management skills and theory. (Meredith \& Mantel, 2005).

- Team-Project Approach - Teams, like any organization, are complex, dynamic and goal-oriented. The team-project or organizational approach focuses on management of the team and the projects stakeholders (Miller, 2009). In virtual or online project team environments, the team-project approach would be considered most suitable due to the fundamental attributes for such an approach with respect to distance of project participants, expected rapid changes and the utmost priority for innovation.

Web-based technologies have "provided us with the tools and incentives that allow us to link individuals and groups across geographic, functional, project and organizational boundaries (Espinosa et al., 2002). However, the complexities of planning and affecting communications considering the growing global nature of our organizations, institutions and social groupings is challenging project planners to reevaluate communications planning approaches (Miller, 2009).

\subsection{Distributing Information}

Firm business relationships are founded on full and open communication between the parties. Collaboration tools such as email, Instant Messaging (IM), websites, and other web-based technologies can assist the project team in performing its information-sharing competence and activities. When communications plans prioritize information-sharing with all members of the team, as well as other key project stakeholders, there is more often than not a demonstrated commitment across the project (Lewis, 2007).

Planning the distributing of information across the project also infers a keen knowledge of how various collaborative technologies are currently utilized and an understanding of how to incorporate various communication technologies, where appropriate. Information distribution in this context includes distribution of both formal and informal information; use of internal project management information systems, use of mobile and fix telephone and teleconferencing technologies; storing templates, project documents and electronic project data; and the establishment and maintenance of data backup procedures.

\subsection{Managing Expectations}

Managing client expectations involves not only identifying the expectation itself but also suggesting ways to gain more control of or influence each expectation. Client's expectations for a given project can affect a wide range of interactions, including service responsiveness, service capability, product functionality, and project success. Furthermore, expectations are difficult to control and impossible to turn off (Karten, 1994).

Current approaches to both identifying and influencing client expectations focus on the need for project planners to focus their attention both to the client's base expectations, the standard by which perceived performance will be evaluated, and to the client's perceptions of actual performance; the overall goal is to educate clients enabling them to make sound judgments about project performance. Two challenging keys to ensuring expectation is met are to make sure that all members of the team speak with one voice and client communications speak the language of the customer (Gray \& Larson, 2008). 


\subsection{Reporting Performance}

Performance reporting involved displaying measurements of actual results of specific project activities over a given time period and compares those results to budgeted or standard measurements obtained under some conditional assumptions over the same period. Any variations or variances are noted and corrective actions are suggested where appropriate. Performance reporting ensures that stakeholders are informed on the use of resources as they are plied to project objectives. Reporting performance can take the form of forecasts, progress and status reports and can be delivered using a wide variety of approaches and media services including both hard and soft copy; synchronous and asynchronous events; reoccurring and by exception delivery; and to internal and external stakeholders.

\section{Incorporating Semantic \& 3D Web into Communication Plans}

When developing communications plans, project leads must view information as not only what can be consumed, but also what its consumers contribute to. Notions of using a mixture of technologies such as semantic and virtual are to foster a shared intelligence (Ollus, Jansson, Karvonen, Uoti, \& RiiKonen, 2009). Information retrieval by semantic web retrieval tools and use of 3D Web environments increases the project team's ability to have valuable data to share amongst project stakeholders; however, there is still room for information gaps and information overload. Project managers must also consider communications channels and their availability, stakeholder relationships, and be aware of the tracking phase of information. Incorporating the use of semantic and 3D Web technologies within project communications planning will see the development of new approaches and methodologies that will view project communications in a whole new light.

\subsection{Identifying Stakeholders}

The continued growth of social networking and demand for user-content generation is forging a new form of web-based relationship building. Studies of social network effects find that web users are influenced directly by the decisions of a typically small subset of other network users. The extent and density of users clustering within these networks, as well as the information that is accessed, becomes strategic in technology adoption and planning (Shuen, 2008).

As noted earlier by their very nature stakeholders are each unique retaining specific roles and responsibilities both on and off the project. Harrison and Lock (2004) noted with concern that the "temporary nature of project organizations allows insufficient time for interpersonal relationships to reach the static state possible in routine operation management". However, the explosion of user-generated content is having a significant impact to the way project planners will be looking to tailor their web-based communications planning efforts. Now there are web-based technologies beginning to take advantage of all of this new Web content. For example, discovery sites like ZoomInfo, Spock, and Rapleaf along with business-focused web-based professional services like LexisNexis, Generate, and ExecRelate are beginning to construct user databases by crawling the Web and taking publically available information on individuals and making associations that map the people and their relationships to others. By automating the collect of this information, these sites are able to create a user map or online social graph throughout the web. This online social graph is for people what the World Wide Web is for hyperlinked web pages allowing for organizing, filtering, and association of information (Shil, 2009).

As semantically enabled content continues to permeate project data repositories, the ability to automate the collection of stakeholder data and the mapping of roles, and relationships that are both formal and personal in nature. Beyond cataloguing each stakeholder's basic information, knowledge of their level of interest and influence with respect to the project will be graphed to better understand each stakeholder, the perspective that they come from, and the relationships that they bring to the project.

Another aspect of stakeholder knowledge is to establish core competencies despite the variety of skill levels possessed by stakeholders at project inception. A semantic-driven tool for data retrieval will help provide the knowledge core and virtual world participation requires a specific skill set especially for real time collaborations. Semantic and virtual world tools would help to manage risk and optimize performance.

Finally, where semantic technologies provide the data, 3D Web environments provide the platform for stakeholder interaction. Unlike the flat 2D Web spaces, 3D Web technologies provide users with rich cognitive cues that enhance the collaboration experience and provide for a real-time sense of presence.

\subsection{Planning Communications}

Planning project communications encompasses defining how the team will manage all aspects of communication. From a project teaming perspective, 3D Web technologies provide members with flexible environments for holding meetings, conducting training and skills development sessions, role-play and develop and present simulations, collect and exhibit libraries and art, support disabilities, provide language and culture training and immersion. Burden (2009) notes that 
these environments are not so much about what they look like but about what is in them; pointing out that what is growing is a continous demand for access and interactions with web content in the form of information and resources that manage and retain that information.

We as humans gain knowledge about our surroundings through our five senses: visual, auditory, kinesthetic, smell, and taste with the first three typically being our primary sensory receptors. Since individuals process information more efficiently when presented in the preferred sensory form it (Lewis, 2007), then it can be to the project team's advantage to understand these preferences and plan their communication modalities accordingly. The 3D Web technologies also provide for browsing of various information spaces, virtual libraries, and other document collections; viewing virtual renditions of various locations (i.e. cities, worksites, campuses, etc.); and navigating through streaming audio/video archives.

The technical ability of stakeholders to transfer, receive, decipher and analyze electronic information is a fundamental need of any workable communication amongst a project team or organization. Project managers must also address issues of need and technical ability of stakeholders at the beginning phase of their communication planning. Full integration of program participants is of high priority. Delaying initial integration of work produced by remote teams can cause significant impact on projects in the form of untimely or delayed task outcomes (Ilincic, 2008).

In addition to technical needs and skill sets is the ability of stakeholders to communicate effectively with one another individually and as a group. Often unsuccessful projects are the result of poor communication skills amongst stakeholders regardless of technical issues. Many virtual teams fail to reach their project objectives because team members fail to communicate with each other (Čulo \& Skendrović, 2009). One approach to minimizing this issue is to quickly devise a communications strategy that takes into account effective ways for dealing with issues of trust, information flow by watching for various communication patterns across the various channels. The use of socially enabled Web spaces can be a polarizing discussion within any team communications planning. In early 2008 the CEO at Serena Software forwarded a memorandum to all 900 of his employees that instituted Facebook Friday in an effort to not only keep his employees in tune with current media technologies but also encourage them to interact and learn from each other (Kirkpatrick, 2008).

It has been well observed that with face-to-face interaction 58 percent of communication is through body language, 35 percent is through how the words are said, and only 7 percent is through the content of words the person is saying (Mehrabian, 1981). By extending media options that incorporate more of the sensory receptors such as 3D Web environments within the communications plan, project leaders can open new and channels that address the needs and sensory preferences of their team members and other key stakeholders.

When planning for incorporation of semantic web technologies, establishment of project-wide lexicons early in the planning process will be critical. Project planners must establish early on in the planning process whether or not they will use their own project lexicon or vocabulary within the organization. They must determine whether to treat their own PM vocabulary as a knowledge base for the organization itself. At the project team level, members can either develop their own vocabulary or make a commitment to use of the client lexicon where applicable. This is especially the case for projects with stakeholders where a global understanding is necessary for effective communication (Delisle \& Olson, 2004). If words like 'success' are to be used for evaluative purposes within the organization then all team members and stakeholders must be onboard (consensus) with its' meaning and share a common concept of the word. In order for project managers to conquer the aspect of the organizational information needs they must have a common semantic base and know all stakeholders involved.

\subsection{Distributing Information}

After communication management plans are designed, the distribution of information could be described as the communication monitoring process. There are several issues within the communication planning phase that have a direct impact on the distribution of information. The first issue addresses the need to establish a level of trust so that information flows along the agreed upon and appropriate lines of communication. Trust between project team and client or between any two or more stakeholders is earned by doing what one says they will do on a continued, repeated basis. Within the information distribution process trust comes from repeatedly receiving and sending project information across various formal and informal channels of communication and thus also infers some level of reliability in the modality as well. The increased usage of Web-based technologies has demonstrated benefits that include better documentation, improved trust and information sharing and sustained growth (Petroske, 2008).

The second issue focuses on ensuring that once trust issues are overcome that information traffic flow is oncoming. Using the traffic analogy, one might consider more cars on the highway as being a negative thing. However, in online 
networks more traffic is a positive. Positive network effects created the online exponential growth of such web-based networks as Google, Yahoo!, eBay, Wikipedia, and Flickr. Positive network effects increase the value of the services provided as more users adopt it (Shuen, 2008). For the project planner this translates to more stakeholder information data flow makes for positive network effect.

Finally, the necessity to control duplication raises a third challenge for the information distributing process. For project planners, duplication of information may provide a challenging three-fold mix. The mixes involved are technological (web-based semantic and virtual tools) empirical (data) and stylistic (leadership) with the latter, a mixture of leadership styles currently deemed the best approach of the three (Thomas \& Bendoly, 2009). Mixed leadership styles are naturally inclined toward mixed technologies. Issues of trust using mixed technologies and leadership style although more relaxed also present some challenges that current practices need to overcome.

Finally, communication plans and distribution modalities must incorporate the issues of accessibility from trusted sources and those sources trusting the retrievers need to be established. Hence, plans must consider that there are within current practices issues of trust and accessibility to data (Gemmill, 2006).

\subsection{Managing Expectations}

The old saying that 'you can't manage what you don't measure' holds true when looking to manage client expectations. Expectations are the driving force behind most client actions and so they become the key measure for any projects success or failure. Establishing, monitoring, and whenever possible, influencing expectations are critical for the successful completion of any project. In the current dynamic global environment expectations of clients tend to be extremely high and are primarily a communication process (Garrett, 2007). Managing client expectations is all about communications, on the front-end of the project, between client and project team regarding what is required of the project.

Project planners must also have a full grasp on the expectations of the technologies within this framework of project communication needs. The attractive nature of new technologies such as $3 \mathrm{D}$ virtual environments have a newness or 'cool factor' about them and the temptation to embrace 3D Web technologies is great. Prior to embracing these technologies project planners should ensure they understand their stakeholder's communications needs and understand how the communities that reside in these virtual environments operate and function. Project managers need to set clear objectives before and during such an undertaking (Gonsalves, 2008).

\subsection{Reporting Performance}

Project performance reporting begins at the work package level where information is gathered regarding the progress of specific deliverables. Here, information is presented as outlined in project plans and often requires visibility of the information across all stakeholders (Morris \& Pinto, 2007). Semantic web technologies are all about making data easier to work with. Business applications are all about the data, and any competitive edge is sought. The web vision for the Semantic Web is at odds with business - the ideals of open data, open source, run counter to business need for security, reliability, and control.

As Semantic Web data becomes more pervasive, it inevitable that companies will pay to have their content ranked higher and found easier that other content. The Semantic Web is a new computer language for describing all the knowledge that people could ever save in books and computers. It lets programmers connect facts and ideas that would otherwise be located in all sorts of different places, making it much easier for people to find things they need even though there is so much information in the world. Currently, Google is limited to helping users find indexed words in documents, whereas the design of the Semantic Web helps people find ideas and concepts in any kind of data.

\section{Assessing the Need or Where's the Win-Win}

As noted earlier, finding and retrieving the best information possible is imperative to any successful communication planning. Given what is at risk due to any level of failure in the communications process, the communication directions inherent in semantic and 3D web technologies offers project planners several win-win solution sets. This is particularly the case if both of these technologies are used in tandem. Each of these technologies addresses specific problems or issues faced within projects; especially those project relaying heavily on remote participants and online communications options.

\subsection{Bridging the Semantic Gap}

Discussions on how best to meet the informational needs in an electronic age have been evolving since the early 1990's. Communications planning addresses potential semantic gaps between project participants and especially for those responsible for retrieving and distributing information. The principle reason for bringing individuals or groups from outside one's community is to bridge knowledge concepts since all have the same or similar goals in meeting research or 
informational needs. Research in the 1990's concluded that having a semantic web-based network to narrow the semantic gap set the groundwork for a more holistic approach to goal setting for everyone involved (DeMichelis et al., 1997).

Similar research efforts pertain to the difficulties surrounding project managers retrieving data only presented in 2D text-based forms. Besides the semantic ambiguities of interdisciplinary information we find project participants also having to have their local (and linked to remote communities) of researchers all looking for information with data in a variety of formats including 3D models. There is also the notion of not only retrieving more graphical data, but compatibility issues for viewing and manipulation follow. To this end, the arrival of new virtual technologies can show much promise in tackling issues with data existing within non-text formats as well as retrieval and manipulation of them in real time (Whyte, Bouchlaghem, Thorpe, \& McCaffer, 2000) (Tay \& Roy, 2003).

\subsection{Common Team Lexicon}

Having the correct lexicon or vocabulary operating from within a team or organization is important and challenging for those remote stakeholders participating. However, having a common vocabulary is only half the battle since terminology and definition may not always be perceived in the same ways. Nailing down the specific meaning or concepts within any discipline in any language is difficult and project management terms included. Operating within the same vocabulary runs far deeper than looking for 'consensus' and observing what people actually use or do not use in practice (Delisle \& Olson, 2004). This addresses the notion of concept and lands us square in the realm of semantic tools. Furthermore, Espinosa et al. discusses the term local context which goes a bit broader than what is associated with language and culture. It also includes other unknowns for project participants such as who and when to call (Espinosa, Cummings, Pearce, \& Wilson, 2002).

Project managers will need to understand the organizational culture includes both lexicon or vocabulary and local context in which they manage. Knowing the organizational culture including those with remote stakeholders helps project managers better assess which types of web application tools to introduce for optimum communication flow. In today's high-tech society and the blend of social networks, the chances are great for mixed technologies including those with semantic-driven knowledge bases.

\subsection{Web Application Hybrids 'Mashups' Lead the Way}

Semantics within the Web represents a clear follow-on to the evolution of mashups. Semantics gives the Web a knowledge representation providing an interface system that combines the rule-based and object-oriented paradigms (Abiteboul, Greenshpan, \& Milo, 2008). These technologies are continually expanding in scope providing more flexibility and scalability for end users. For project managers, operational procedures for all stakeholders should strongly consider adding semantic and virtual tools to both communication planning efforts. To be successful at information integration, information gatherers must begin to incorporate web application hybrids or mashups. Recent history suggests mashups prove to be the direction toward future project management efforts (Kloppenborg, 2008).

Mashups, a term borrowed from the precursors of music sampling, have had a powerful influence over today's market of web users. Mashup technologies on the web are the layering of other technical existing tools and services upon one another resulting in a powerful comprehensive deployment of information and resources all viewed in one place (Abiteboul, Greenshpan, \& Milo, 2008). Tools such as Flicker, E-bay and Google are 'mashed' together in one site creating a portal-like web resource. Mashups are created in order to combine powerful web-based technologies and overlaying them with different sets of data in such a way that the information derived from such a fusion allows for more effective analysis (Lake, 2006). It appears that mashups are expanding in number for a variety of reasons beyond their use to inform the general public. The use of mashups is on an upward trend with development-oriented technology-based corporations like Google, wanting to help users create and design even more creative mashups in order to promote their own agendas (Lake, 2006).

\subsection{Web 2.0 Technologies Create Social Interaction}

With the advent of Web 2.0 technologies including 2D and 3D, distance of participants for project planners with regard to communication is less of an issue than in the past. Granted that regardless of the geographic distance there is technical compatibility, stakeholders are able to communicate in synchronous and asynchronous ways. Drawing semantic data to control for any local context issues and manipulation of that data or objects in real time is now made possible with the each of these technologies operating as a combined force. Now place the semantic and virtual tools with other layers of the Web 2.0 technologies and the way communication plans are drawn by project managers of today is in stark contrast with those practices two decades ago. The limitations of geographic boundaries of the past are now becoming non-existent in an electrically entrenched world. Today, there are boundaries of a different kind. 
Another term is 'functional boundary' (Espinosa, Cummings, Pearce, \& Wilson, 2002), mentioned in reference to research problems when analyzing teams. Functional boundaries strikes directly at proximity issues in distance and time, which in virtual 3D worlds would be 'virtually-speaking', potentially made moot. Undoubtedly, virtual 3D world use for project management changes the landscape of communication planning. New approaches are being introduced with many more on the horizon.

\section{Impact Summary}

Current planning practices in managing communications can be extended into mixed technological platforms; however, this still requires setting proper expectations and clear objectives for the technologies involved. Given the nature of virtual teams and their round-the-clock accessibility to all stakeholders, creating semantically enabled project repositories is critical for efficient work. Harris et. al., (2008) summed this up when discussing a recent virtual team; "this virtual team operates 24/7/365 and it all hinges on standards, measures and processes for success. There's little time to learn on the job." (Harris, Herron, \& Iwanicki, 2008)

In accordance with this paper's premise, other authors have also reached similar conclusions regarding the coming demand for both technologies. There will be strong demand for intelligent tools that combine web and database content with greater precision; these tools of productivity will enable 'procedural generation' referring to dynamically generated content on the fly rather than prior to distribution (Zaboura, 2009). The attractive data sets would definitely be those offered within a 3D Web framework. Other predictions include one by a Gartner researcher, Adam Sarner who indicated that within the next five years that time spent online will significantly compete with time spent in real time (Sarner, 2007). This would validate predictions that project planners will look to spend much of their time dealing with stakeholders who are doing the same.

Use of semantic and virtual 3D Web technologies lends itself to creating a better environment to foster collaboration thereby improving communication amongst team members and project stakeholders. The end result for project planners with regard to semantic and 3D Web is that these tools of technology are being intertwined with the cultural or societal shifts in this century. In order for project management success, both cultural and technological shifts are not to be viewed as mutually exclusive.

\section{References}

Abiteboul, S., Greenshpan, O., \& Milo, T. (2008). Modeling the Mashup Space. 10th ACM International Workshop on Web Information and Data Management (WIDM). Napa Valley, CA: ACM. http://dx.doi.org/10.1145/1458502.1458517

Agarwal, A. (2009). Web 3.0 Concepts Explained in Plain English. Retrieved Sept 7, 2009, from Digital Inspiration: http://www.labnol.org/internet/web-3-concepts-explained/8908/

Burden, D. (2009). Toward Semantic Virtual Worlds - A Thinkpiece. Retrieved September 3, 2009, from www.converj.com: http://www.converj.com/sites/converjed/2009/05/toward_semantic_virtual_worlds.html

Čulo, K., \& Skendrović, V. (2009). Paradigms of virtual teams in the communication process. Informatologia, 42(3), 197-204.

Delisle, C. L., \& Olson, D. (2004). Would the real project management language please stand up? International Journal of Project Management, 22(4) , 327-337. http://dx.doi.org/10.1016/S0263-7863(03)00072-3

DeMichelis, G., Dubois, E., Jarke, M., Mylopoulos, J., Papazoglou, M., Pohl, K., et al. (1997). Cooperative Information Systems: A Manifesto. Trends \& Directions, 1-37.

Espinosa, J. A., Cummings, J. N., Pearce, B. M., \& Wilson, J. M. (2002). Research on teams with multiple boundaries. Proceedings of the 35th Annual Hawaii International Conference on System Sciences (pp. 3429- 3438). Honolulu, HI: IEEE Explore. http://dx.doi.org/10.1109/HICSS.2002.994381

Farr, C., \& Irias, X. (2008). Communication specialists and Engineers: Partners in Successful Project Delivery. Proceedings of the Water Environment Federation, The Utility Management Conference 2008 (pp. 408-417). Chicago, IL: Water Environment Federation. http://dx.doi.org/10.2175/193864708788805486

Garrett, G. A. (2007). World Class Contracting, 4th Ed. Riverwoods, IL: Wolters Kluwer.

Gemmill, J. (2006). A Trust-Relationship Management Framework for Federated Virtual Organizations. Birmingham, AL: Purushotham V. Bangalore. 
Gonsalves, A. (2008). Most Business-Launched Virtual Worlds Fail, Gartner Says. Retrieved November 20, 2009, fromInformationWeek: http:/www.informationweek.com/news/personal_tech/virtualworlds/showArticle.jhtml? articleID $=207800625$

Gray, C. F., \& Larson, E. W. (2008). Project Management: The Mangerial Process. New York, NY: McGraw-Hill.

Harris, M. D., Herron, D., \& Iwanicki, S. 2008). The Business Value of IT: Managing Risks, Optimizing Performance and Measuring Results. Boca Raton, FL: Auerbach Publications. http://dx.doi.org/10.1201/9781420064759

Harrison, F. L., \& Lock, D. (2004). Advanced Project Management: A Structured Approach, 4th Ed. Burlington, VT: Gower.

Ilincic, R. (2008). Examining Agile Management Methods and Non-Agile Management Methods in Global Software Development Projects. Boston. MA: Northeastern.

K Zero. (2009). K Zero: 260M Registered Accounts for the 10-15-Year-Old Virtual World Demo. Retrieved September 16, 2009, from VirtualWorldNews.com: http://www.virtualworldsnews.com/2009/02/k-zero-260m-registered-accounts-for-the-1015yearold-virtual-world-d emo.html

Karten, N. (1994). Managing Expectations: Working with People Who Want More, Better, Faster, Sooner, Now! . New York, NY: Dorset House Publishing.

Kerzner, H. (2006). Project Management: A Systems Approach to Planning, Scheduling, and Controlling, 9th ed. Hoboken, NJ: John Wiley \& Sons.

Kirkpatrick, D. (2008). Fortune Magazine. Retrieved November 12, 2009, from money.cnn.com: http://money.cnn.com/2008/06/12/technology/kirkpatrick_facebook.fortune/index.htm

Kloppenborg, T. J. (2008). Contemporary Project Managment. New York, NY: South Western Educational Publishing.

Lake, N. (2006). Mashups: Melting Pots of Search Tools. Plugged In , 47-49.

Lewis, J. P. (2007). The Project Manager's Desk Reference, 3rd Ed. New York, NY: McGraw-Hill.

Masinsin, R. Q. (2008). Secretary of Defense Corporate Fellows Program: Final Report. New York, NY: Time Warner/CNNMoney.

McDavid, D. (2008). Sociable Technologies for Enterprising Sociality. Proceedings of the 52nd Annual Meeting of the ISSS (pp. 1-32). Elk Grove, CA: International Society for the Systems Sciences.

Mehrabian, A. (1981). Silent Messages: Implicit Communication of Emotions and Attitudes, 2nd Ed. Belmont, CA: Wadsworth.

Meredith, J. R., \& Mantel, S. J. (2005). Project Management: A Managerial Approach. Hobocken, NJ: John Wiley \& Sons.

Miller, K. (2009). Organizational Communications: Approaches and Processes. Boston, MA: Wadsworth Publishing.

Mitham, N. (2009). Growth forecasts for the Virtual Worlds sector. Retrieved September 16, 2009, from www.kzero.co.uk: http://www.kzero.co.uk/blog/?m=200909

Morris, P. W., \& Pinto, J. K. (2007). The Wiley Guide to Project Control. Hoboken, NJ: John Wiley \& Sons.

Nicholas, J. \& Steyn, H. (2008). Project Management for Business, Engineering, and Technology: Principles and Practice. Burlington, MA: Butterworth-Heinemann.

Ollus, M., Jansson, K., Karvonen, I., Uoti, M., \& RiiKonen, H. (2009). On Services for Collaborative Project Managment. In L. M. Camarinha-Matos, I. Parackakis, \& H. Afsarmanesh, Leveraging Knowledge for Innovation in Collaborative Networks (pp. 451-462). Thessaloniki, Greece: 10th IFIP WG 5.5 Working Conference on Virtual Enterprises, PRO-VE 2009. http://dx.doi.org/10.1007/978-3-642-04568-4_47

O'Reilly, T. (2005). What Is Web 2.0: Design Patterns and Business Models for the Next Generation of Software. Retrieved Sept 7, 2009, from O'Reilly: http://oreilly.com/web2/archive/what-is-web-20.html

Petroske, J. (2008). Promoting Project Communications Using Wikis. 4th International Project Management Conference. Anchorage, AK: ProMAC.

Phillips, J. (2006). Project Management Professional Study Guide, Second Edition. San Francisco, CA: McGraw-Hill. 
Sarner, A. (2007). Generation $V$ will have a profound influence on culture, society and business, says Gartner. Retrieved November 23, 2009, from Terrati.com: http://software.tekrati.com/research/9692/

Schwalbe, K. (2010). Information Technology Project Managment 6e. Boston, MA: Course Technology - Cengage Learning.

Shil, C. (2009). The Facebook Era: Tapping Online Social Networks to Better Products, Research new Audiences and Sell More Stuff. New York, NY: Prentice Hall.

Shuen, A. (2008). Web 2.0: A Strategy Guide. Sebastopol, CA: O'Reilly.

Strickland, J. (2009). Is there a Web 1.0? Retrieved Sept 6, 2009, from computer.howstuffworks.com: http://computer.howstuffworks.com/web-10.htm

Tay, F. E., \& Roy, A. (2003). CyberCAD: a collaborative approach in 3D-CAD technology in a multimedia-supported environment . Computers in Industry, 52(2), 127-145. http://dx.doi.org/10.1016/S0166-3615(03)00100-3

Thomas, D., \& Bendoly, E. (2009). Limits to Effective Leadership Style and Tactics in Critical Incident Interventions. Project Mangement Journal, 40(2) , 70-80. http://dx.doi.org/10.1002/pmj.20118

Virtual World Review. (2009). What is a Virtual World?. Retrieved Sept 10, 2009, from Virtual Worlds Review: http://www.virtualworldsreview.com/info/whatis.shtml

Whyte, J., Bouchlaghem, A., Thorpe, A., \& McCaffer, R. (2000). From CAD to virtual reality: modelling approaches, data exchange and interactive 3D building design tools. Automation in Construction, 10(1) , 43-55. http://dx.doi.org/10.1016/S0926-5805(99)00012-6

Zaboura, N. (2009). CReATE - Creating a Joint Research Agenda for Promoting ICT-Innovations in Creative Industries across Europe. Stuttgart, Germany: MFG Baden-Wuerttemberg mbH, Public Innovation Agency for ICT and Media. Retrieved Sept 6, 2009, from lets-create.eu: http://www.lets-create.eu/ fileadmin/_create/downloads/BGP_ICT_and_Creative_Industries_2008.pdf 